\title{
MAPPING THE VIRGO CLUSTER OF GALAXIES WITH ASCA
}

T. OHASHI, K. KIKUCHI, K. MATSUSHITA, N. Y. YAMASAKI,

A. KUSHINO AND ASCA VIRGO PROJECT TEAM

Department of Physics, Tokyo Metropolitan University

1-1 Minami-Ohsawa, Hachioji, Tokyo 192-03, Japan

\section{Introduction}

In the Virgo cluster, we can perform a close study of the gas injection mechanism from galaxies into the cluster space and the interaction between the injected gas and the sorrouding cluster medium. In 1996 to 1997, we carried out mapping observations of a $2 .^{\circ} 5 \times 2 .^{\circ} 5$ area in the north-west region of the cluster. There are 16 pointings in total in this region, and the observed results are briefly reported here.

\section{Observed Results}

The mosaic map of the GIS image (Kikuchi et al. 1997) shows enhanced Xray emission from bright galaxies. To look into a large-scale distribution of the ICM properties, we examined GIS spectra in each pointed region with $40^{\prime}$ diameter. Since the cluster emission is only $20-30 \%$ brighter than the diffuse X-ray background in the outermost regions ( $>3^{\circ}$ offset from M87), selection of the background affects the results significantly. Because of the long-term increase of the GIS non X-ray background by about $5 \% \mathrm{yr}^{-1}$ (Ishisaki 1997), we used black sky data taken in 1997 for the background.

The pulse-height spectra corrected for the background are fitted with thermal models by Raymond and Smith. Figure 1(a) and (b) show distribution of the ICM temprature and metal abundance as a function of the distance from M87. The error bars indicate $90 \%$ limits allowing for a $\pm 10 \%$ fluctuation of the diffuse background intensity. As seen clearly, the temprature shows a systematic drop with radius and becomes $50-60 \%$ of the central level at $r \sim 1 \mathrm{Mpc}$. The results are not yet corrected for the stray light which tends to smear the temperature gradient. 
The metal abundance shows a narrow peak centered at M87 (Matsumoto et al. 1996) and then shows a gradual drop in large scale. It is $0.2-0.3$ solar within $400 \mathrm{kpc}$ from M87 and less than 0.2 solar at $r>600$ kpc. Note that the abundance at this temperature is mainly detemined by Fe-L lines, for which discrepancy exists among theoretical models.

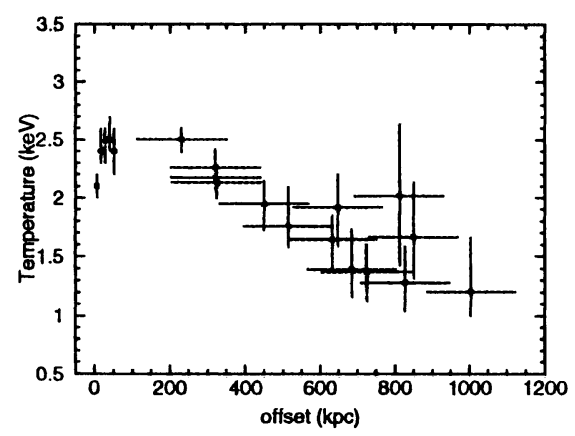

Figure 1. ICM Temprature as a function of distance from M87

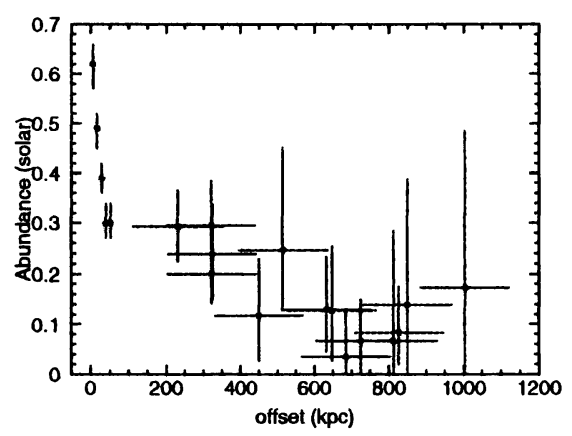

Figure 2. Distribution of metal abundance

\section{Discussion}

The temperature drop in the outer region of clusters is predicted from numerical simulations (e.g. Eke et al. 1997), and recently observed as an average properties of about 30 clusters by Markevitch (1997). If temerature gradient is steeper in clusters which are in the early stage of gravitational collapse (i.e. outer region is not heated enough), this would be an additional indication that the Virgo cluster is a young system (Böhringer et al. 1994). It is intersting that other systems such as AWM7 and Perseus cluster show more uniform temperature distribution than the Virgo cluster.

The abundance gradient is roughly consistent with the galaxy distribution as already seen in AWM7 (Ezawa et al. 1997). This suggests that metals $(\mathrm{Fe})$ are mostly injected from the present population of galaxies and no strong mixing has occured in the ICM.

\section{References}

Böhringer, H. et al. (1994) Nature, 368, 828

Eke et al. (1997) submitted to $A p J$, (astro-ph/9708070)

Ezawa et al. (1997) ApJ Letters, Nov. 20 in press

Ishisaki, Y. (1997) Ph. D. Thesis, University of Tokyo (ISAS RN-613)

Kikuchi, K. et al. (1997) ASCA/ROSAT Workshop on Clusters of Galaxies, p. 221

Markevitch, M. (1997) in preparation

Matsumoto, H. et al. (1996) PASJ, 48, 201 